
coco e pó de rocha. Horticultura Brasileira 26: 499-503.

\title{
Produção de mudas de tomateiro em substratos contendo fibra de coco e pó de rocha
}

\author{
Regynaldo A Sampaio; Sílvio J Ramos; Denílson O Guilherme; Cândido A da Costa; Luiz Arnaldo \\ Fernandes
}

UFMG-ICA, Av. Universitária, s/nº, Bairro Universitário, C. Postal 135, 39404-006 Montes Claros-MG; rsampaio@ufmg.br

\begin{abstract}
RESUMO
Com o objetivo de avaliar proporções dos resíduos fibra de coco (FC) e pó de rocha de granito (RG) como substratos na produção de mudas de tomateiro, cultivar Kada Gigante, realizou-se um experimento em casa de vegetação na UFMG-ICA. Foi utilizado o delineamento experimental em blocos ao acaso, com quatro repetições. Os tratamentos foram constituídos por uma testemunha e seis proporções de fibra de coco (FC) e pó de rocha de granito (RG): $\mathrm{T}_{1}$ Substrato comercial Hortimix ${ }^{\circledR} ; \mathrm{T}_{2}-0 \% \mathrm{FC}+100 \% \mathrm{RG} ; \mathrm{T}_{3}-20 \%$ $\mathrm{FC}+80 \% \mathrm{RG} ; \mathrm{T}_{4}-40 \% \mathrm{FC}+60 \% \mathrm{RG} ; \mathrm{T}_{5}-60 \% \mathrm{FC}+40 \% \mathrm{RG} ; \mathrm{T}_{6}$ $-80 \% \mathrm{FC}+20 \% \mathrm{RG} ; \mathrm{T}_{7}-100 \% \mathrm{FC}+0 \% \mathrm{RG}$. O cultivo foi feito em bandeja de poliestireno expandido com células de 3,5 x 3,5 x 5,0 $\mathrm{cm}$. Foram avaliados o diâmetro do coleto, a altura da planta, o índice de velocidade de emergência, a percentagem de emergência e a massa fresca e seca da parte aérea e da raiz. Em praticamente todas as características avaliadas o substrato comercial foi superior aos substratos contendo fibra de coco e pó de rocha. Considerando-se apenas os substratos contendo fibra de coco e pó de rocha, as mudas com melhores características foram obtidas com a mistura de aproximadamente $70 \%$ em volume de fibra de coco.
\end{abstract}

Palavras-chave: Lycopersicon esculentum, resíduo de marmoraria, reciclagem.

\begin{abstract}
Tomato seedlings production using substrates with coconut fiber and rock waste

A greenhouse experiment was carried out to evaluate the ratio of coconut fiber and rock waste, in the tomato seedling production (cultivar Kada Gigante), in Montes Claros, Brazil. The experimental design was of randomized complete blocks with four replicates. The treatments were composed of a control and six ratios of coconut fiber (FC) and rock waste (RG): $\mathrm{T}_{1}-$ Commercial Substrate Hortimix $^{\circledR} ; \mathrm{T}_{2}-0 \% \mathrm{FC}+100 \% \mathrm{RG} ; \mathrm{T}_{3}-20 \% \mathrm{FC}+80 \% \mathrm{RG} ; \mathrm{T}_{4}-$ $40 \% \mathrm{FC}+60 \% \mathrm{RG} ; \mathrm{T}_{5}-60 \% \mathrm{FC}+40 \% \mathrm{RG} ; \mathrm{T}_{6}-80 \% \mathrm{FC}+20 \%$ $\mathrm{RG} ; \mathrm{T}_{7}-100 \% \mathrm{FC}+0 \% \mathrm{RG}$. The cultivation was done in polystyrene trays with cells of $3.5 \times 3.5 \times 5,0 \mathrm{~cm}$. Stem diameter, seedling height, emergency speed index, emergency percentage, root and aerial part of fresh and dry matter were evaluated. In almost all the evaluated characteristics the commercial substrate was better than the substrates containing coconut fiber and rock waste. Among the substrates containing coconut fiber and rock waste, the seedlings with better characteristics were obtained with a mixture of approximately $70 \%$ of the volume of coconut fiber.
\end{abstract}

Keywords: Lycopersicum esculentum, marble residues, recycling.

\section{(Recebido para publicação em 16 de abril de 2007; aceito em 31 de outubro de 2008) (Received in April 16, 2008; accepted in October 31, 2008)}

$\mathrm{N}$ tentativa de se encontrar soluções para minimizar o impacto ambiental causado pelo descarte de resíduos urbanos e industriais, como casca de arroz, bagaço de cana, casca de pinus, lixo e resíduo da produção de papel, vários segmentos da sociedade têm se empenhado no desenvolvimento de pesquisas que visam o aproveitamento econômico desses materiais, os quais muitas vezes apresentam potencial para o aproveitamento agrícola, principalmente como substratos na produção de mudas (Chong, 1999; Backes \& Kämpf, 1991; Flynn et al., 1995; Souza, 2001; Sainju et al., 2001).

A utilização de resíduos da agroindústria local como componentes para substratos pode propiciar a redução dos custos na produção de mudas, assim como auxiliar na minimização de impactos ambientais negativos (Fermino, 1996; Silveira et al., 2002). Porém, é importante lembrar que os substratos devem apresentar boa capacidade de troca catiônica, estabilidade física e esterilidade biológica e adequados $\mathrm{pH}$, condutividade elétrica, teor de nutrientes, relação $\mathrm{C} / \mathrm{N}$, relação água/ar, porosidade total, capacidade de retenção de água e drenagem (Lemaire, 1995; Sassaki, 1997; Borne, 1999; Konduru et al., 1999; Booman, 2000; Carrijo et al., 2004).

Com a restrição da utilização do xaxim e da turfa, uma alternativa é a utilização de matérias primas regionais, como a fibra de coco e o pó de rocha de granito, que são de fácil obtenção (Theodoro, 2000; Rosa et al., 2001b; Arenas et al., 2002). Segundo Carrijo et al. (2002), as boas propriedades físicas da fibra de coco, a sua não reação com os nutrientes da adubação e longa durabilidade sem alteração de suas características físicas, assim como a abundância da matéria prima que é renovável e o baixo custo para o produtor, fazem da fibra de coco verde um substrato dificilmente superável por outro tipo de substrato, mineral ou orgânico no cultivo sem solo de hortaliças e flores. Também, Rosa et al. (2001a) destacam que o pó de coco verde é um meio de cultivo $100 \%$ natural e pode ser indicado para germinação de sementes, propagação de plantas em viveiros e no cultivo de flores e hortaliças.

Diante do exposto, o objetivo deste trabalho foi avaliar a potencialidade da utilização hortícola do pó de rocha originado da serragem de granito, juntamente com a fibra de coco, na produção de mudas de tomate.

\section{MATERIAL E MÉTODOS}

$\mathrm{O}$ experimento foi conduzido em casa de vegetação na UFMG-ICA, em Montes Claros-MG, utilizando-se a cul- 
Tabela 1. Características químicas dos materiais utilizados na produção dos substratos para a produção de mudas de tomateiro (chemical characteristics of the materials used in the production of substrates for the production of tomato seedlings). Montes Claros, UFMG, 2005.

\begin{tabular}{|c|c|c|c|}
\hline Determinação & $\begin{array}{l}\text { Substrato } \\
\text { comercial }\end{array}$ & $\begin{array}{c}\text { Fibra de } \\
\text { coco }\end{array}$ & $\begin{array}{l}\text { Pó de } \\
\text { rocha }\end{array}$ \\
\hline Matéria Orgânica ${ }^{1}\left(\mathrm{~g} / \mathrm{dm}^{3}\right)$ & 22,60 & 24,77 & 3,39 \\
\hline pH em água & 6,0 & 6,6 & 7,5 \\
\hline $\mathrm{N}$ total $^{2}(\mathrm{dag} / \mathrm{kg})$ & 1,83 & 0,56 & 0,25 \\
\hline P disponível ${ }^{3}$ (dag/kg) & 0,92 & 0,15 & 0,03 \\
\hline $\mathrm{K}_{3}(\mathrm{dag} / \mathrm{kg})$ & 0,39 & 1,15 & 0,20 \\
\hline $\mathrm{Ca}_{4}(\mathrm{dag} / \mathrm{kg})$ & 0,24 & 0,45 & 4,62 \\
\hline $\mathrm{Mg}_{4}(\mathrm{dag} / \mathrm{kg})$ & 0,89 & 0,20 & 0,31 \\
\hline $\mathrm{S}(\mathrm{dag} / \mathrm{kg})$ & 0,03 & 0,02 & 0,70 \\
\hline $\mathrm{Zn}_{5}(\mathrm{mg} / \mathrm{kg})$ & 16,1 & 12 & 36 \\
\hline $\mathrm{Cu}_{5}(\mathrm{mg} / \mathrm{kg})$ & 14,5 & 0,3 & 20,4 \\
\hline $\mathrm{Mn}(\mathrm{mg} / \mathrm{kg})$ & 45,1 & 14 & 12 \\
\hline Densidade $\left(\mathrm{kg} \cdot \mathrm{dm}^{-3}\right)$ & 0,47 & 0,98 & 1,02 \\
\hline Capacidade de Retenção de Água (g H20. g ${ }^{-1}$ substrato) & 2,7 & 3,0 & 0,57 \\
\hline C.E. ${ }_{6}(\mathrm{mS} / \mathrm{cm})$ & 1,80 & 2,18 & 189,35 \\
\hline
\end{tabular}

${ }^{1}$ Método da perda por irrigação (Kiehl, 1985) (method for the loss irrigation (Kiehl, 1985)); ${ }^{2}$ Método Micro-Kjeldahl (Kiehl, 1985) ( ${ }^{2}$ Micro-Kjeldahl method (Kiehl, 1985)); ${ }^{3}$ Extrator Mehlich-1 (Defelipo \& Ribeiro, 1981) (Mehlich-1 extractor (Defelipo \& Ribeiro, 1981)); ${ }^{4}$ Extrator KCL $1 \mathrm{~mol} / \mathrm{L}$ (Defelipo \& Ribeiro, 1981) $\left({ }^{4} \mathrm{KCl} 1 \mathrm{~mol} / \mathrm{L}\right.$ extractor (Defelipo \& Ribeiro, 1981)); ${ }^{5}$ Extrator $\mathrm{HNO}_{3} / \mathrm{HClO}_{4}$ (Tedesco et al., 1995) $\left(\mathrm{HNO}_{3} / \mathrm{HClO}_{4}\right.$ extractor (Tedesco et al., 1995)); ${ }^{6}$ Extrato de saturação na relação 1:5 (Tedesco et al., 1995) (saturation extract in a relation 1:5 (Tedesco et al., 1995)).

tivar de tomate, Kada Gigante, de 15/ 01 a $14 / 02 / 05$.

Os tratamentos foram constituídos por uma testemunha e seis proporções, com base no volume, de fibra de coco verde (FC) e pó de rocha de granito $(\mathrm{RG})$ : $\mathrm{T}_{\mathbf{1}}$ - Substrato comercial Hortimix $^{\circledR} ; \mathrm{T}_{2}-0 \% \mathrm{FC}+100 \%$ $\mathrm{RG} ; \mathrm{T}_{3}-20 \% \mathrm{FC}+80 \% \mathrm{RG} ; \mathrm{T}_{4}-40 \% \mathrm{FC}$ $+60 \% \mathrm{RG} ; \mathrm{T}_{5}-60 \% \mathrm{FC}+40 \% \mathrm{RG} ; \mathrm{T}_{6}-$
$80 \% \mathrm{FC}+20 \% \mathrm{RG} ; \mathrm{T}_{7}-100 \% \mathrm{FC}+0 \%$ RG. As características químicas e físicas dos materiais usados na produção dos substratos encontram-se na Tabela 1.

A fibra de coco verde foi seca ao ar durante uma semana e processada em desintegrador da marca Cremasco ${ }^{\circledR}$ linha DPCT.4 com peneira de $10 \mathrm{~mm}$. A quantidade de fibra adicionada aos tratamentos foi calculada com base no volume.

O semeio manual foi feito colocando-se uma semente no centro de cada uma das 128 células da bandeja de poliestireno expandido de 3,5 x 3,5 x $5,0 \mathrm{~cm}$. O delineamento experimental foi o de blocos casualizados, com quatro repetições. A unidade experimental foi constituída por 16 plantas, sendo avaliados o diâmetro do coleto (D), a altura da planta (A), o índice de velocidade de emergência (IVE), a percentagem de emergência (E), a massa fresca da parte aérea (MFPA) e da raiz (MFSR), e, a massa seca da parte aérea (MSPA) e da raiz (MSSR). A emergência foi avaliada 20 dias após a semeadura, considerando-se emergidas as sementes que emitiram o caulículo. A altura da planta, medida do coleto até o ápice da muda, e o diâmetro do coleto, foram avaliados aos 28 dias após a semeadura. O índice de velocidade de emergência foi quantificado, utilizandose o método de Maguire (1962), de acordo com a fórmula IVE. $=\Sigma(\mathrm{Pi} / \mathrm{Di}), \mathrm{em}$ que: IVE $=$ índice de velocidade de emergência; $\mathrm{Pi}=$ número de plântulas emergidas no i-ésimo dia de contagem; Di $=$ número de dias que as plântulas levaram para emergir no i-ésimo dia de contagem.

Os dados obtidos foram submetidos à análise de variância e as médias das proporções de fibra de coco e de pó de rocha de granito foram comparadas com

Tabela 2. Valores médios obtidos de percentagem de emergência (E), índice de velocidade de emergência (IVE), diâmetro do coleto (D), altura da planta (A), massa fresca sistema radicular (MFSR), massa seca sistema radicular (MSSR), massa fresca parte aérea (MFPA) e massa seca parte aérea (MSPA), em plântulas de tomate, cultivar Kada Gigante, produzidas em diferentes substratos. (average values of percentage of emergency (E), emergency speed Index (IVE), collect diameter (D), height of the plant (A), fresh matter of roots (MFSR), dry matter of roots (MSSR), fresh shoots (MFPA) and shoot dry matter (MSPA) in seedlings of tomato, cultivar Kada Gigante, produced on different substrates). Montes Claros, UFMG, 2005.

\begin{tabular}{|c|c|c|c|c|c|c|c|c|}
\hline \multirow{2}{*}{ Substratos } & \multirow{2}{*}{$\begin{array}{c}E \\
(\%) \\
\end{array}$} & \multirow{2}{*}{ IVE } & \multirow{2}{*}{$\begin{array}{c}D \\
(\mathrm{~mm})\end{array}$} & \multirow{2}{*}{$\begin{array}{c}A \\
(\mathrm{~cm})\end{array}$} & MFSR & MSSR & MFPA & MSPA \\
\hline & & & & & \multicolumn{4}{|c|}{ (g) } \\
\hline $0 \% \mathrm{FC}+100 \% \mathrm{RG}$ & $29,70^{*}$ & $0,55^{\star}$ & $0,78^{*}$ & $2,18^{*}$ & $0,0173^{*}$ & $0,0026^{*}$ & $0,0188^{*}$ & $0,0041^{*}$ \\
\hline $20 \% \mathrm{FC}+80 \% \mathrm{RG}$ & $65,60^{*}$ & $1,70^{*}$ & $0,84^{*}$ & $2,42^{*}$ & $0,0276^{*}$ & $0,0024^{*}$ & $0,0341^{*}$ & $0,0048^{*}$ \\
\hline $40 \% \mathrm{FC}+60 \% \mathrm{RG}$ & $86,90^{\text {ns }}$ & $2,32^{*}$ & $0,74^{*}$ & $2,61^{*}$ & $0,0304^{*}$ & $0,0020^{*}$ & $0,0483^{*}$ & $0,0053^{*}$ \\
\hline $60 \% \mathrm{FC}+40 \% \mathrm{RG}$ & $90,10^{\text {ns }}$ & $2,39^{*}$ & $0,81^{*}$ & $2,53^{*}$ & $0,0238^{*}$ & $0,0022^{*}$ & $0,0257^{*}$ & $0,0047^{*}$ \\
\hline $80 \% \mathrm{FC}+20 \% \mathrm{RG}$ & $81,70^{\text {ns }}$ & $2,12^{*}$ & $0,84^{*}$ & $2,22^{*}$ & $0,0258^{*}$ & $0,0035^{*}$ & $0,0469^{*}$ & $0,0054^{*}$ \\
\hline $100 \% \mathrm{FC}+0 \% \mathrm{RG}$ & $86,40^{\mathrm{ns}}$ & $2,23^{*}$ & $0,99^{*}$ & $2,37^{*}$ & $0,0155^{*}$ & $0,0033^{*}$ & $0,0329^{*}$ & $0,0050^{*}$ \\
\hline Substrato Comercial & 97,90 & 3,17 & 2,01 & 8,82 & 0,8375 & 0,0286 & 0,8868 & 0,1149 \\
\hline (DMS) & 25,33 & 0,75 & 0,38 & 0,73 & 0,065 & 0,020 & 0,107 & 0,011 \\
\hline
\end{tabular}

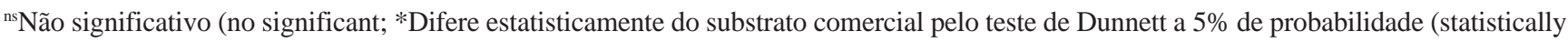
differs from commercial substrate by Dunnett test at $5 \%$ probability); FC = Fibra de coco $(\mathrm{FC}=$ coconut fiber); $\mathrm{RG}=\mathrm{Pó}$ de rocha de granito $(\mathrm{RG}=$ Rock waste). 


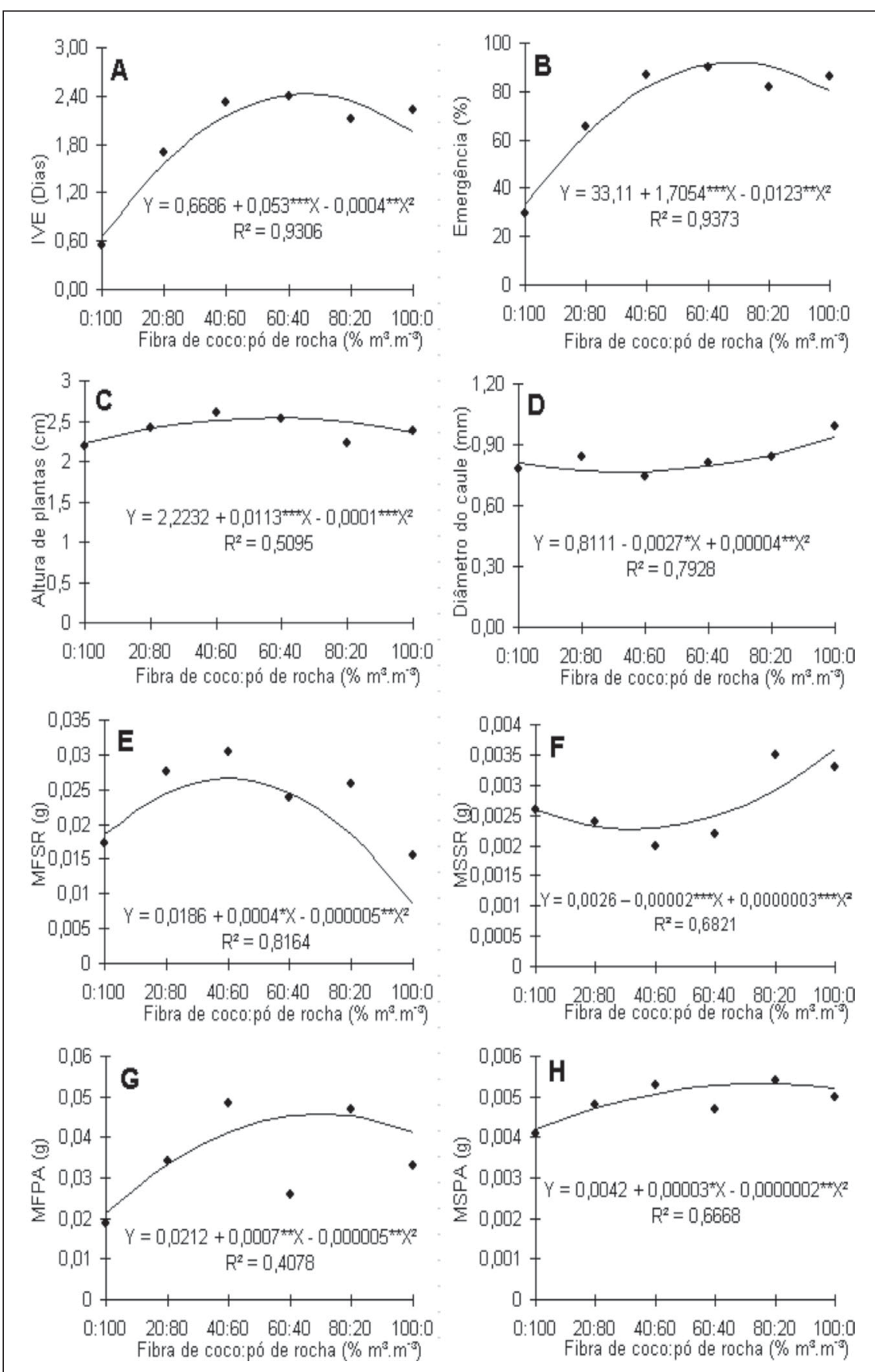

Figura 1. Índice de velocidade de emergência (A), percentagem de emergência (B), altura da planta (C), diâmetro do coleto (D), massa fresca (E) e seca (F) da raiz e massa fresca (G) e seca $(\mathrm{H})$ da parte aérea de mudas de tomateiro em função da proporção de fibra de coco e pó de rocha no substrato. (index speed emergency (A), percentage of emergency (B), plant height (C), diameter of collect (D), weight (E) and dry (F) and the root weight (G) and dry $(\mathrm{H})$ of shoot of tomato seedlings according to the proportion of coconut fiber and powder of rock from the substrate). Montes Claros, UFMG, 2005.

$*, * *, * * *$ Significativos a $5 ; 1$ e $0,1 \%$ de probabilidade, respectivamente, pelo teste t. significant at $5 ; 1$ and $0.1 \%$ probability, respectively, by the $t$ test).

as do substrato comercial até 5\% de probabilidade pelo teste de Dunnett. As médias referentes às proporções de fibra de coco e de pó de rocha de granito foram ajustadas a modelos de regressão até maiores valores de índice de velocidade de emergência, diâmetro do coleto, altura da planta, massa fresca e seca da raiz e massa fresca e seca da parte aérea, em relação aos substratos contendo fibra de coco e/ou pó de rocha de granito (Tabela 2). Tal fato pode ser atribuído à menor densidade e maiores teores de nitrogênio e fósforo do substrato comercial (Tabela 1), favorecendo a aeração e respiração das raízes, e maior nutrição, vigor e crescimento das plantas. Rodrigues (1994) afirma que mudas de tomateiro para processamento industrial têm melhor desempenho em substratos que apresentam boas condições de aeração e de armazenamento de água. Silveira et al. (2002), Oliveira et al. (2004) e Costa et al. (2007), estudando o desenvolvimento de tomate em substratos comerciais e alternativos, destacam melhor desempenho dos substratos comerciais em razão de suas melhores características de retenção de água, aeração e teores de nutrientes. Também, Arenas et al., (2002) constataram que mudas de tomate crescidas em substratos contendo mais de $50 \%$ de pó de coco não se desenvolveram tão vigorosamente quanto aquelas desenvolvidas em turfa, mesmo quando fertilizadas duas vezes semanalmente com $50 \mathrm{mg} / \mathrm{L}$ de nitrogênio. Os autores atribuem tal fato à imobilização do nitrogênio disponível pelos microrganismos, em razão da elevada relação $\mathrm{C} / \mathrm{N}$ deste resíduo.

Por outro lado, o substrato comercial apresentou a mesma percentagem de emergência dos substratos contendo $40 \%$ ou mais de fibra de coco (Tabela 2). Neste caso, o aumento na quantidade de fibra de coco em relação ao pó de rocha pode ter proporcionado maior aumento na retenção de umidade do substrato (Tabela 1), favorecendo diretamente a emergência das sementes. A necessidade de uma adequada retenção de umidade do substrato em relação à emergência de sementes e crescimento das plantas tem sido apontada por Lemaire (1995) e Fernández-Bravo et al. (2006), principalmente para volume de substrato inferior a $2 \mathrm{~L}$. Arenas et al. (2002), estudando o uso de perlita em comparação a turfa, fibra de coco e vermiculita como substratos para a produção de mudas de tomateiro, verifica- 
ram que a perlita atrasou a germinação das sementes em razão da sua menor retenção de água. Não obstante, Silveira et al. (2002) observaram que a fibra de coco proporcionou maior percentagem de emergência de plântulas de tomateiro $(90,63 \%)$ do que o substrato comercial $(67,18 \%)$, porém, as mudas nele produzidas foram menos vigorosas do que no substrato comercial, sendo esse problema corrigido somente quando o pó de coco foi misturado ao húmus de minhoca.

Houve influência das diferentes proporções de fibra de coco e de pó de rocha sobre as características das mudas. O índice de velocidade de emergência apresentou ajuste quadrático em relação à mistura de fibra de coco e pó de rocha, com destaque para o maior índice com $66,25 \%$ de fibra de coco na mistura (Figura 1A). A percentagem de emergência das sementes apresentou também comportamento quadrático. Constatou-se, com a relação de 69,32\% de fibra de coco em relação ao pó de rocha de granito, um percentual máximo de emergência das sementes de 92,22\% (Figura 1B). Valores maiores de pó de rocha em relação à fibra de coco na composição dos substratos proporcionaram baixos índices de velocidade de emergência, além de menor desenvolvimento das mudas. Tal fato pode estar relacionado à redução da aeração do substrato proporcionado pelo aumento da proporção do pó de rocha, conforme discutido por Lemaire (1995), o qual aponta que as partículas finas podem preencher os espaços vazios e alterar a geometria do espaço poroso, afetando a circulação de ar e de água no substrato. Também, Minami (1995) cita que o substrato é o componente mais sensível e complexo do sistema de produção de mudas, sendo que qualquer variação na sua composição altera o processo final de produção de mudas, desde a não germinação de sementes até o desenvolvimento irregular das plantas.

A altura das mudas de tomateiro em relação à mistura da fibra de coco com pó de rocha foi quadrática (Figura 1C), obtendo-se altura máxima de $2,54 \mathrm{~cm}$ com $56,5 \%$ de fibra de coco na mistura. Essa foi a melhor relação entre fibra de coco e o pó de rocha de granito, possi- velmente por ter proporcionado melhores condições de retenção de água e suprimento de nutrientes para as plantas. $\mathrm{O}$ efeito positivo do uso de substratos contendo fibra de coco na produção de mudas de tomate, possivelmente associado a uma maior capacidade de disponibilidade de nutrientes e água, também foi observado por Carrijo et al. (2004).

$\mathrm{O}$ diâmetro do coleto apresentou um comportamento quadrático com o aumento da fibra de coco na mistura com pó de rocha, observando-se tendência de diâmetro mínimo de $0,76 \mathrm{~mm}$ na relação de $33,75 \%$ de fibra coco. Valores acima de $33,75 \%$ de fibra de coco promoveram aumento deste diâmetro (Figura 1D). Esta superioridade da fibra de coco em relação ao desenvolvimento das mudas pode ser explicada pela maior retenção de umidade e maiores teores de nitrogênio total e fósforo disponível neste material, conforme verificado na Tabela 1. Além disso, a elevada salinidade do pó de rocha, caracterizada pela sua condutividade elétrica, pode ter contribuído para baixar o potencial de água no substrato nas maiores doses, afetando a absorção de água pelas plantas. Também, o pH em torno de 7,5, pode ter reduzido a disponibilidade dos micronutrientes para as plantas (Lemaire, 1995).

As massas fresca e seca do sistema radicular apresentaram ajuste quadrático em relação às diferentes proporções de fibra de coco e pó de rocha. Os maiores valores destas variáveis foram, respectivamente, 0,026 e 0,0022 g, obtidos com 40 e $100 \%$ de fibra de coco em relação ao pó de rocha (Figuras 1E e 1F). Ficou evidente, portanto, o aumento da massa seca da raiz com o incremento da proporção de fibra de coco em relação ao pó de rocha. Tal fato pode estar associado ao aumento da retenção de umidade do substrato, conforme já discutido. De acordo com Sturion (1981) o substrato exerce uma influência marcante sobre o sistema radicular, atribuído principalmente à quantidade e tamanho das partículas que definem a aeração e a retenção de água necessários ao crescimento das raízes.

Com relação às massas fresca e seca da parte aérea, os resultados expressaram resposta quadrática. Verificou-se que os maiores valores obtidos para es- tas variáveis foram, respectivamente, 0,45 e $0,0053 \mathrm{~g}$, nas proporções 70 e $75 \%$ de fibra de coco, respectivamente (Figuras 1G e 1H). Constatou-se um aumento do crescimento e, conseqüentemente, das massas fresca e seca da parte aérea, onde a proporção de fibra de coco foi maior que a do pó de rocha na constituição do substrato. Tal fato pode ser atribuído à maior capacidade de retenção de água deste resíduo, como verificada por Pragana (1998), proporcionando uma condição favorável para o crescimento de mudas de tomateiro.

Neste estudo, verificou-se que o substrato comercial proporcionou condições adequadas ao crescimento das plântulas de tomateiro, haja vista a maior produção de massa fresca e seca da parte aérea e do sistema radicular das mesmas. Por outro lado, ficou evidente que o pó de rocha de granito ou a fibra de coco, quando usados puros, não são substratos ideais para a produção de mudas de tomateiro, já que as plântulas se desenvolveram melhor com a mistura dos dois em proporções contendo em torno de $70 \%$ de fibra de coco. Segundo Silveira et al. (2002) o pó de coco puro não revelou ser um bom substrato para a produção de mudas, uma vez que as plântulas não apresentaram bom desenvolvimento. Para ser eficiente como substrato esse material deverá ser empregado em mistura com outros materiais mais ricos em nutrientes.

Diante do exposto, os resultados sugerem que a fibra de coco pode ser um componente fundamental na mistura de substratos recomendados para a produção de mudas de tomateiro.

\section{REFERÊNCIAS}

ARENAS M; VAVRINA CS; CORNELL JA; HANTON EA; HOCHMUTH GJ. 2002. Coir as an alternative to peat in media for tomato transplant production. HortScience 37: 309312.

BACKES MA; KÄMPF AN. 1991. Substratos à base de composto de lixo urbano para a produção de plantas ornamentais. Pesquisa Agropecuária Brasileira 26: 753-758.

BOOMAN JLE. 2000. Evolução dos substratos usados em horticultura ornamental na Califórnia. In: KÄMPF AN; FERMINO MH. (Eds) Substratos para plantas: a base da produção vegetal em recipientes. Porto Alegre: Gênesis, p. 43-65.

BORNE HR. 1999. Produção de mudas de 
hortaliças. Guaíba: Agropecuária, 189p.

CARRIJO OA; LIZ RS; MAKISHIMA N. 2002. Fibra da casca do coco verde como substrato agrícola. Horticultura Brasileira 20: 533-535.

CARRIJO OA; VIDAL MC; REIS NVB; SOUZA RB; MAKISHIMA N. 2004. Produtividade do tomateiro em diferentes substratos e modelos de casas de vegetação. Horticultura Brasileira 22: 05-09.

CHONG C. 1999. Experiences with the utilization of wastes in nursery potting mixes and field soil amendments. Canadian Journal of Plant Science 79: 139-148.

COSTA CA; RAMOS SJ; SAMPAIO RA; GUILHERME DO; FERNANDES LA. 2007. Fibra de coco e resíduo de algodão para substrato de mudas de tomateiro. Horticultura Brasileira 25: 387-391.

DEFELIPO BV; RIBEIRO AC. 1981. Análise química do solo: metodologia. Viçosa: UFV, 17p. (Boletim de extensão 29).

FERMINO MH. 1996. Aproveitamento de resíduos industriais e agrícolas como alternativas de substratos hortícolas. Porto Alegre: UFRGS, 90p. (Tese mestrado).

FERNANDEZ-BRAVO C; URDANETA N; SILVA W; POLISZUK H; MARÍN M. 2006. Germinación de semillas de tomate (Lycopersicon esculentum Mill.) cv Río Grande sembradas en bandejas plásticas, utilizando distintos sustratos. Revista de La Faculdad de Agronomia 23: 188-196.

FLYNN RP; WOOD CW; GUERTAL EA. 1995. Lettuce response to composted broiler litter as a potting substrate component. Journal of American Society for Horticultural Science 6:
964-970.

KIEHL EJ. 1985. Fertilizantes orgânicos. São Paulo: Ceres. 492p.

KONDURU S; EVANS MR; STAMPS RH. 1999. Coconut husk and processing effects on chemical and physical properties of coconut coir dust. HortScience 34: 88-90.

LEMAIRE F. 1995. Physical, chemical and biological properties of growing medium. Acta Horticulturae 396: 273-284.

MAGUIRE JD. 1962. Speed of germination: aid in selection and evaluation for seedling emergence and vigor. Crop Science 2: 176-177.

MINAMI K. 1995. Fisiologia da produção de mudas. São Paulo: T.A. Queiroz. 129p.

OLIVEIRA GB; MOTA WF; MAGALHÃES VR; ALVES FG; SILVEIRA EKCP; TARCHETTI GP. 2004. Produção de mudas de tomateiro rasteiro com a utilização de substratos alternativos ao produto comercialmente utilizado. In. CONGRESSO BRASILEIRO DE OLERICULTURA, 44. Resumos... Campo Grande: SOB (CD-ROM).

PRAGANA RB. 1998. Potencial do resíduo da extração da fibra de coco como substrato na produção agrícola. Recife: UFRPE. 84p. (Tese mestrado).

RODRIGUES AG. 1994. Sistema de produção para a cultura do tomate industrial no norte de Minas Gerais. Belo Horizonte: EPAMIG, 5p. (Boletim técnico 42).

ROSA MF; ABREU FAP; FURTADO, AAL; BRÍGIDO, A.K.L.; NORÕES ERV. 2001a. Processo agroindustrial: obtenção de pó de casca de coco verde. Fortaleza: Embrapa Agroindústria Tropical. 4p. (Comunicado técnico 61).

ROSA MF; SANTOS FJS; MONTENEGRO AAT; ABREU FAP; CORREIA D; ARAUJO FBS; NORÕES ERV. 2001b. Caracterização do pó da casca de coco verde usado como substrato agrícola. Fortaleza: Embrapa Agroindústria Tropical. 6p. (Comunicado técnico 54).

SAINJU UM; RAHMAN S; SINGH BP. 2001. Evaluating hairy vetch residue as nitrogen fertilizer for tomato in soilless medium. HortScience 36: 90-93.

SASSAKI OK. 1997. Resultados preliminares da produção de hortaliças sem o uso de solo no Amazonas. Horticultura brasileira 15: 165169.

SILVEIRA EB; RODRIGUES VJLB; GOMES AMA; MARIANO RLR; MESQUITA JCP. 2002. Pó de coco como substrato para produção de mudas de tomateiro. Horticultura Brasileira 20: 211-216.

SOUZA FX. 2001. Materiais para formulação de substratos na produção de mudas e no cultivo de plantas envasadas. Fortaleza: EmbrapaCNPAT. 21p. (Documentos 43).

STURION JA. 1981. Método de produção e técnicas de manejo que influenciam o padrão de qualidade de mudas de essências florestais. Curitiba: Embrapa-URPFCS. 5p. (Documentos 3).

TEDESCO MJ; GIANELLO C; BISSANI CA; BOHNEN H; VOLKWEISS SJ. 1995. Análise de solo, plantas e outros materiais. UFRGS: Porto Alegre. 174p.

THEODORO SCH. 2000. A fertilização da terra pela terra: uma alternativa para sustentabilidade do pequeno produtor rural. Brasília: UNB. 225p. (Tese doutorado). 